\title{
Constitutive expression of IRF-5 in HTLV-1-infected T cells
}

\author{
CHIE ISHIKAWA $^{1,4}$, MASACHIKA SENBA ${ }^{2}$, BETSY J. BARNES ${ }^{3}$ and NAOKI MORI ${ }^{4}$ \\ ${ }^{1}$ Transdisciplinary Research Organization for Subtropics and Island Studies, University of the Ryukyus, \\ Nishihara, Okinawa 903-0213; ${ }^{2}$ Department of Pathology, Institute of Tropical Medicine, Nagasaki University, \\ Nagasaki 852-8523, Japan; ${ }^{3}$ Department of Microbiology, Biochemistry and Molecular Genetics, \\ Rutgers Biomedical and Health Sciences, Newark, NJ 07103, USA; ${ }^{4}$ Department of Microbiology and Oncology, \\ Graduate School of Medicine, University of the Ryukyus, Nishihara, Okinawa 903-0215, Japan
}

Received January 30, 2015; Accepted March 16, 2015

DOI: 10.3892/ijo.2015.3020

\begin{abstract}
Human T-cell leukemia virus type 1 (HTLV-1) is the etiologic agent of adult T-cell leukemia (ATL), an aggressive and fatal leukemia of $\mathrm{T}$ cells. Interferon regulatory factor (IRF)-5 plays a critical role in the induction of interferon genes in viral infected cells. We examined the specific mechanisms underlying the expression and regulation of IRF-5 in HTLV1-infected T cells. IRF-5 was constitutively transcribed into three distinct alternatively spliced isoforms (V1, V3 and V4) in HTLV-1-infected T-cell lines but not in uninfected T-cell lines. IRF-5 was also upregulated in HTLV-1-infected T-cell lines at protein level. Nuclear IRF-5 expression was noted in ATL cells present in lymph nodes and skin lesions. IRF-5 mRNA expression was induced following infection of T cells with HTLV-1, and specifically by viral oncoprotein Tax. Tax also activated V3 promoter. Microarray analysis of IRF-5-expressing uninfected $T$ cells demonstrated that IRF-5 induced the expression of tumor necrosis factor family cytokines. The results suggest that IRF-5 is a Tax-regulated gene, and its expression may be associated with the pathogenesis of ATL.
\end{abstract}

\section{Introduction}

Interferon regulatory factor (IRF)-5 is a transcription factor member of the IRF family that regulates the expression of genes induced by viral infection (1). While IRF-5 was identified as a regulator of type I interferon (IFN) (2), further studies indicated that IRF-5 plays essential roles in the regulation of

Correspondence to: Professor Naoki Mori, Department of Microbiology and Oncology, Graduate School of Medicine, University of the Ryukyus, 207 Uehara, Nishihara, Okinawa 903-0215, Japan E-mail: naokimori50@gmail.com

Dr Chie Ishikawa, Transdisciplinary Research Organization for Subtropics and Island Studies, University of the Ryukyus, 1 Senbaru, Nishihara, Okinawa 903-0213, Japan

E-mail: cheizo@lab.u-ryukyu.ac.jp

Key words: human T-cell leukemia virus type 1, adult T-cell leukemia, Tax, interferon regulatory factor-5, tumor necrosis factor genes involved in the stimulation of the immune system, cell growth, apoptosis and oncogenesis (3-6). Overexpression of IRF-5 has been associated with autoimmune diseases such as systemic lupus erythematosus and rheumatoid arthritis $(7,8)$. IRF-5 is a direct target of p53 (9) and displays some tumor suppressor properties as it can induce p21, Bak, Bax and caspase- 8 genes $(6,10)$. Downregulation of IRF-5 by hypermethylation has been reported in hepatocellular carcinoma and gastric cancer $(11,12)$. In contrast, IRF-5 is upregulated in thyroid cancer where it contributes to cell proliferation and survival (13). High-level expression of IRF-5 has been also detected in Hodgkin's lymphoma cells and considered to be crucial for their survival (14).

Human T-cell leukemia virus type 1 (HTLV-1) causes either adult T-cell leukemia (ATL) or chronic inflammatory disorders, such as HTLV-1-associated myelopathy/tropical spastic paraparesis, uveitis and arthritis $(15,16)$. The HTLV-1 genome encodes the transactivator Tax protein that plays essential regulatory roles in $\mathrm{HTLV}-1$ replication and oncogenic transformation of $\mathrm{T}$ lymphocytes $(17,18)$. Tax modulates the activation of host signaling pathways to mediate cellular transformation and hyperstimulates the immune system $(17,18)$. Therefore, viral Tax protein is considered to initiate the ATL-related leukemogenesis process, which subsequently progresses towards the ultimate leukemic stage through additional events occurring in Tax absence. In addition, Tax plays a central role in the pathophysiology of chronic inflammatory disorders (19).

With regard to the IRF family of transcription factors, several novel putative targets for Tax-mediated gene activation have been identified. IRF-3, a transcription factor critical in innate immunity to viral infection, is constitutively activated in a Tax-dependent manner (20). On the contrary, oncogenic IRF-4, another member in the IRF family of transcription factors, is overexpressed in lymphocytes of patients with ATL and HTLV-1-transformed T cells (21-25). Tax also induces the expression of IRF-4 (21,25-27). However, the expression of IRF-5 and related regulatory mechanisms have not been fully determined in HTLV-1-infected T cells. In this study, we determined the expression level of IRF-5 in HTLV-1-infected T cells. The results showed that IRF-5 expression was induced by Tax and that it regulated the expression of tumor necrosis factor (TNF) family cytokines. 


\section{Materials and methods}

Cell culture. The HTLV-1-infected MT-2, MT-4, C5/MJ, SLB-1, HUT-102, MT-1, TL-OmI and ED-40515(-) T-cell lines, and the negative control uninfected human leukemia Jurkat, MOLT-4 and CCRF-CEM T-cell lines, were grown in Roswell Park Memorial Institute-1640 medium supplemented with $10 \%$ heat-inactivated fetal bovine serum and antibiotics. JPX-9 cells are derivatives of Jurkat with Tax gene, which is stably integrated under the control of a metallothionein promoter (28). To induce Tax expression, JPX-9 cells were cultured in the presence of $20 \mu \mathrm{M} \mathrm{CdCl}_{2}$. The human acute monocytic leukemia cell line, THP-1, was set as a positive control for IRF-5 expression. TY8-3/MT-2 was established from the interleukin (IL)-2-dependent human T-cell line, TY8-3, co-cultured with mitomycin C (MMC)-treated MT-2 cells, and was capable of growth completely independent of IL-2 (29). Jurkat cells were stimulated with $1,000 \mathrm{U} / \mathrm{ml}$ of recombinant human IFN- $\alpha$ for the indicated time intervals.

HTLV-1 infection by co-cultivation. Peripheral blood mononuclear cells (PBMC) from a healthy donor were isolated from the heparinized blood sample by centrifugation over a Ficoll-Paque layer (GE Healthcare Bio-Sciences AB, Uppsala, Sweden). MT-2 cells were pretreated with $200 \mu \mathrm{g} / \mathrm{ml}$ of MMC for $60 \mathrm{~min}$, pipetted vigorously, and washed three times with phosphate-buffered saline. PBMC and MMC-treated MT-2 cells were co-cultured in the presence of $10 \mathrm{ng} / \mathrm{ml}$ of IL-2. The culture medium was half-changed with fresh medium supplemented with IL-2 every 3 days. Since MT-2 cells were pretreated extensively with MMC, no discernible MT-2 cells were found.

$R T$-PCR. Total RNA was extracted from cells with TRIzol (Invitrogen Life Technologies, Carlsbad, CA, USA) according to the protocol provided by the manufacturer. The RNA was reverse transcribed into cDNA using a PrimeScript ${ }^{\mathrm{TM}}$ RT-PCR kit (Takara Bio Inc., Otsu, Japan). The sequences of the primers for IRF-5, IFN- $\alpha$, IFN- $\beta$, IFN- $\gamma, 2$ ',5'-oligoadenylate synthetase (2-5 AS), MxA, TNF- $\alpha$, lymphotoxin (LT)- $\beta$, $\beta$-actin, glyceraldehyde-3-phosphate dehydrogenase (GAPDH), Tax in HTLV-1-infected T cells, and Tax in JPX-9 cells are summarized in Table I (2,30-36).

Protein extraction and immunoblot analysis. Immunoblot analysis was performed on whole cell lysate, and nuclear and cytoplasmic fractions. Each protein was extracted as previously described (37-39), and subjected to sodium dodecyl sulfate-polyacrylamide gels and transferred to polyvinylidene difluoride membranes. The membranes were then probed for IRF-5 (Abnova Corp., Taipei, Taiwan), actin (NeoMarkers Inc., Fremont, CA, USA), lamin B (Santa Cruz Biotechnology Inc., Santa Cruz, CA, USA) or Myc-Tag (Wako Pure Chemical Industries, Ltd., Osaka, Japan). Mouse monoclonal antibody to Tax, Lt-4, was previously described (40). The bands were visualized using enhanced chemiluminescence kit (Amersham Biosciences Corp., Piscataway, NJ, USA).

Immunofluorescence assays. Cells were fixed in $4 \%$ paraformaldehyde and permeabilized with Triton X-100. Then, the cells were stained with mouse anti-IRF-5 antibody (Abnova
Corp.). For immunofluorescence studies, washed cells were incubated with anti-mouse secondary antibody conjugated with Alexa Fluor 488 (Invitrogen Life Technologies). The nuclei were stained with Hoechst 33342 (Wako Pure Chemical Industries, Ltd.). After final washing, the cells were examined under a Leica DMI6000 microscope (Leica Microsystems, Wetzlar, Germany). Mounted coverslips were viewed through a $63 \mathrm{x}$ oil immersion lens (NA1.4) on a Leica TCS confocal system.

Immunohistochemical analysis. The diagnosis of ATL was based on clinical features, hematological findings and the presence of anti-HTLV-1 antibodies in the serum. Biopsy samples were taken from the lesional skin and lymph nodes of four patients with ATL. IRF-5 immunohistochemistry was performed using an anti-IRF-5 antibody (Abnova Corp.) after pretreatment of the deparafinized tissue sections with ready-to-use proteinase K (Dako, Carpinteria, CA, USA). The sections were counterstained with methyl green, hydrated in ethanol, cleaned in xylene, and mounted. The stained cells were examined under a light microscope (Axicoskop 2 Plus) with an Achroplan 40x/0.65 lens (both from Zeiss, Jena, Germany). Images were acquired with an AxioCam MRc camera and AxioVision 4.7 software (Zeiss). A signed consent form was obtained from each tissue donor.

Plasmids. The IRF-5 P-V3 promoter fragment was cloned into the KpnI and XhoI sites of pGL3-Basic vector (Promega Corp., Madison, WI, USA) (30). Details of the plasmid expressing the HTLV-1 Tax through $\beta$-actin promoter were published previously (41). The coding sequences of IRF-5 were cloned into the pcDNA4/myc-His A vector (Invitrogen Life Technologies).

Transfection and luciferase assay. Jurkat cells were transfected at $5 \times 10^{6}$ cells with the luciferase reporter plasmid, together with $0.5-5 \mu \mathrm{g}$ of the Tax expression vector by electroporation $(250 \mathrm{~V}, 960 \mu \mathrm{F})$. Each transfection included the phRL-TK plasmid (Promega Corp.) as an internal control for variation in transfection efficiency. After $48 \mathrm{~h}$, transfected cells were collected by centrifugation, washed with phosphate-buffered saline, and lysed in reporter lysis buffer (Promega Corp.). The luciferase activity was determined by the Dual-Luciferase Reporter system (Promega Corp.) using the protocol supplied by the manufacturer.

Microarray analysis. Jurkat cells were transfected with a control pcDNA4/myc-His A vector or IRF-5 expression vector using MicroPorator MP-100 (Digital Bio Technology Co., Ltd., Seoul, Korea), pulsed three times at 1,325 V for $10 \mathrm{msec}$ each. After $24 \mathrm{~h}$, total RNA samples were prepared using the RNeasy Plus Mini kit (Qiagen, Hilden, Germany) and confirmed to be of good quality by Agilent 2100 Bioanalyzer (Agilent Technologies, Inc., Waldbronn, Germany). Microarray analysis using a SurePrint G3 Human GE 8x60 K Microarray kit version 2.0 (Agilent Technologies, Inc.) was performed as previously described (42).

\section{Results}

Expression of IRF-5 in HTLV-1-infected T-cell lines. To investigate IRF-5 expression in both HTLV-1-infected and 
A

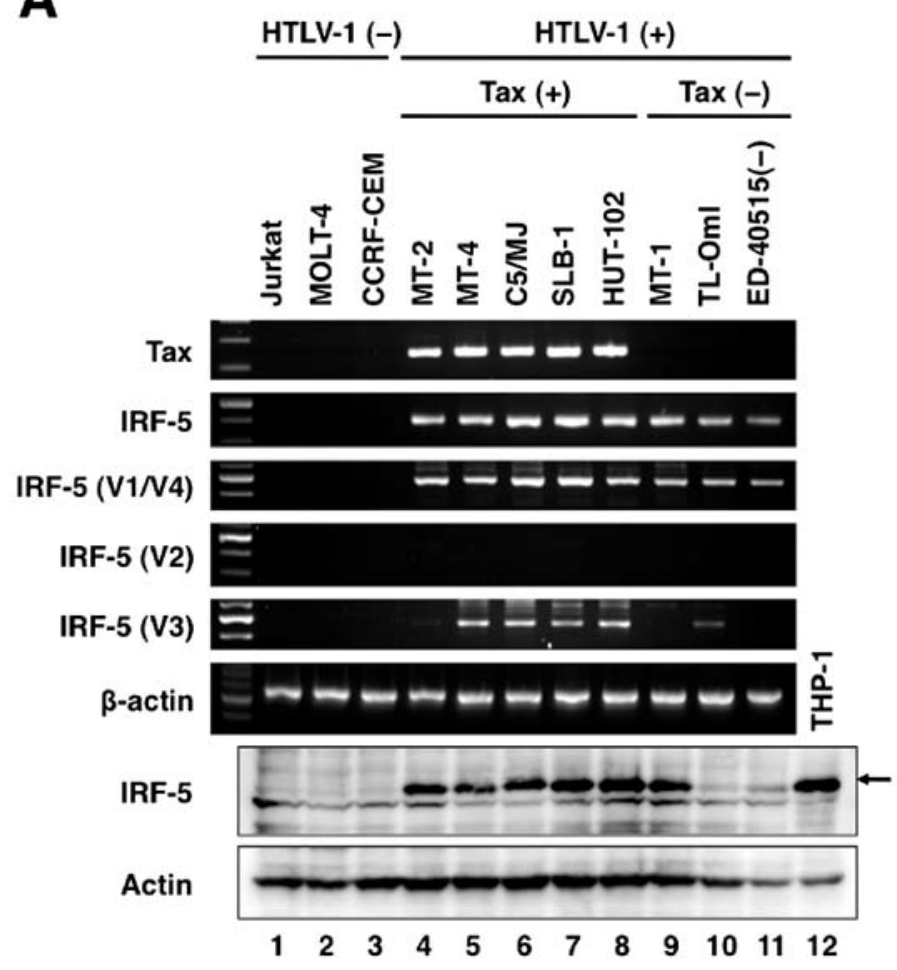

B

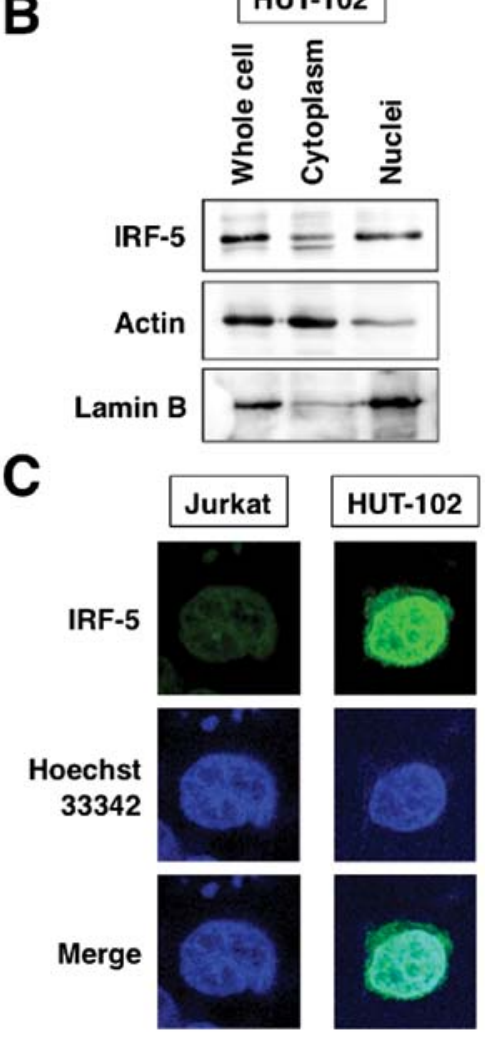

Figure 1. IRF-5 is expressed in HTLV-1-infected T-cell lines. (A) RT-PCR analysis of IRF-5 isoform expression and western blot analysis of IRF-5 expression in uninfected T-cell lines (lanes 1-3), HTLV-1-transformed T-cell lines (lanes 4-8) and ATL-derived T-cell lines (lanes 9-11). RT-PCR was performed with exon 1 isoform-specific primers that amplify through exon 4 (panels 3-5). Expression of $\beta$-actin was used as a control. Lysates from each sample were subjected to immunoblot using an anti-IRF-5 antibody (panel 7). Membranes were reprobed with the anti-actin antibody as a loading control (panel 8). THP-1 was used as positive control. (B) IRF-5 is localized primarily in the nuclei of HTLV-1-infected T cells. Whole cell lysate, and nuclear and cytoplasmic fractions were extracted and analyzed by immunoblotting for IRF-5. Actin and lamin B were used as quality controls to assess cytoplasmic and nuclear fraction purity and loading levels. (C) Jurkat and HUT-102 cells were subjected to immunofluorescence for IRF-5 using the secondary antibody conjugated with Alexa Fluor 488 (green). Hoechst 33342 (blue) was employed to identify the nuclei. The cells were visualized by confocal microscopy.

-uninfected T-cell lines, we collected three uninfected (Jurkat, MOLT-4 and CCRF-CEM), five HTLV-1-transformed (MT-2, MT-4, C5/MJ, SLB-1 and HUT-102) and three ATL-derived [MT-1, TL-OmI and ED-40515(-)] T-cell lines. All HTLV-1transformed T-cell lines constitutively expressed Tax mRNA (Fig. 1A, panel 1). Expression of IRF-5 mRNA in 11 T-cell lines was analyzed by reverse-transcription polymerase chain reaction (RT-PCR) using IRF-5 specific primers. All eight of the HTLV-1-positive T-cell lines examined (lanes 4-11) strongly expressed IRF-5 mRNA, whereas the three HTLV-1-negative T-cell lines (lanes 1-3) did not (Fig. 1A, panel 2). IRF-5 is transcribed into various distinct alternatively spliced isoforms (30), and the IRF-5 variant 1 (V1), V2 and V3 transcripts have different noncoding first exons, whereas V1 and V4 share the same first exon. Primer sets that specifically recognize exon 1 of each isoform (Ex1V1, Ex1V2 and Ex1V3) and a common region in exon 4 of IRF-5 were optimized (Table I).

PCR amplification using the exon 1-specific sense primers was isoform-specific. We next examined the levels of constitutive exon 1-specific IRF-5 isoform expression in several T-cell lines (Fig. 1A, panels 3-5). Ex1V1 transcripts were detected in all HTLV-1-infected T-cell lines (Fig. 1A, panel 3). In comparison, transcripts associated with Ex1V2 could not be detected (Fig. 1A, panel 4). IRF-5 V3 transcript levels were specifically high in Tax-positive HTLV-1-transformed T-cell lines (lanes 5-8), excluding MT-2, suggesting a possible role for Tax in enhanced IRF-5 Ex1V3 transcript levels (Fig. 1A, panel 5). High level of IRF-5 protein expression was evident in Tax-positive HTLV-1-transformed T-cell lines and ATL-derived MT-1 (Fig. 1A, panel 7, lanes 4-9). Intracellular mapping by immunoblotting and immunofluorescence staining showed high levels of IRF-5 in the nuclei of HTLV-1-infected HUT-102 cells (Fig. 1B and C). In contrast, almost no IRF-5 was detected in uninfected Jurkat cells (Fig. 1C).

Abundant IRF-5 expression in ATL cells in lymph nodes and skin lesions. Immunohistochemical staining of ATL cells in archived lymph nodes and skin tissue samples showed abundant IRF-5 in the nuclei of these cells (Fig. 2).

IRF-5 expression during HTLV-1 infection. To examine whether HTLV-1 infection induces IRF-5 expression, studies were performed using the parental TY8-3 cell line and TY8-3 cells infected with HTLV-1 (TY8-3/MT-2). TY8-3/MT-2 cells strongly expressed Tax mRNA (Fig. 3A). RT-PCR analysis also demonstrated upregulation of IRF-5 mRNA in TY8-3/MT-2 cells. To substantiate HTLV-1 control of IRF-5 expression in PBMC, we co-cultured PBMC and MMC-treated MT-2 cells. At seven days after co-cultivation, PBMC were harvested for assessment of expression of HTLV-1 viral gene by RT-PCR. 


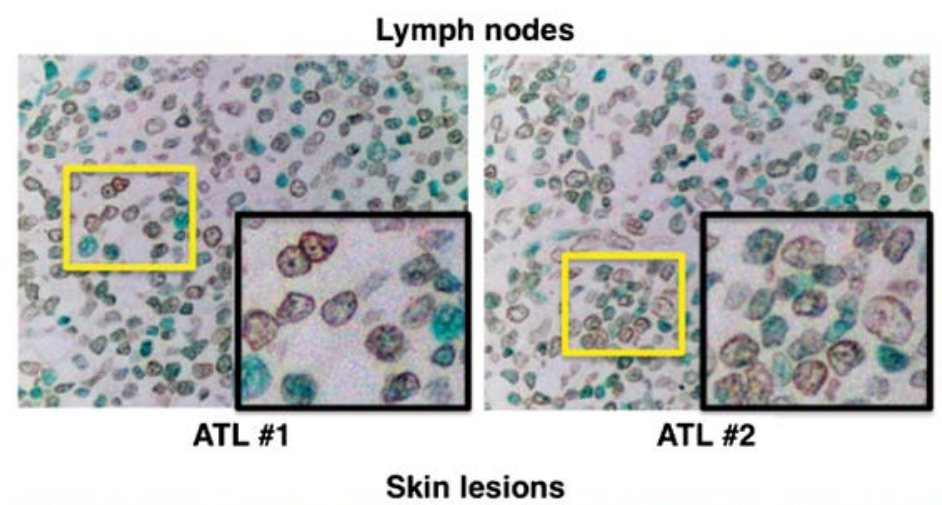

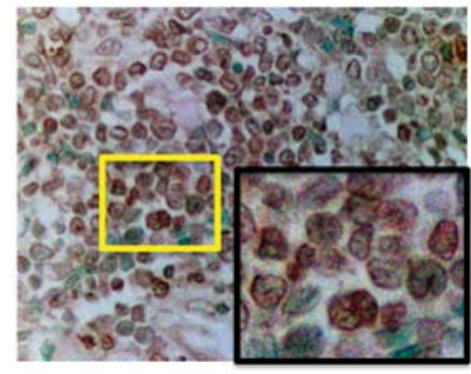

ATL \#3

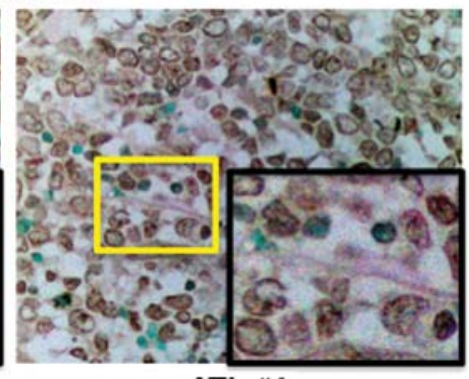

ATL \#4

Figure 2. Representative results of immunohistochemical staining of IRF-5 in ATL lymph nodes and skin lesions. Tissue biopsy sections from ATL lymph nodes and skin lesions were stained with anti-IRF-5 antibody. Tissue sections were counterstained using methyl green. Original magnification, x400. Inset represents higher magnification of the small boxed region (original magnification, $\mathrm{x} 1,000$ ).

Table I. Primer sequences used in RT-PCR.

\begin{tabular}{lll}
\hline Name & \multicolumn{1}{c}{ Forward (5') } & \multicolumn{1}{c}{ Reverse (3') } \\
\hline IRF-5 & GCCTTGTTATTGCATGCCAGC & AGACCAAGCTTTTCAGCCTGG \\
IRF-5 (V1/4) & CCTGGCGCAGCCACGCAGGCGCA & CCAAAAGAGTAATCCTCAGGG \\
IRF-5 (V2) & GCGCCTGGAAAGCGAGCTCG & CCAAAAGAGTAATCCTCAGGG \\
IRF-5 (V3) & CTAGGCAGGTGCAACCCCAAAA & CCAAAAGAGTAATCCTCAGGG \\
IFN- $\alpha$ & CAGGAGGAGTTTGATGGCAACCAG & GACAACCTCCCAGGCACAAGGGC \\
IFN- $\beta$ & ATGACCAACAAGTGTCTCCTCCAAA & GTTTCGGAGGTAACCTGTAAGTCTG \\
IFN- $\gamma$ & ATGAAATATACAAGTTATATCTTGGCTTT & GATGCTCTTCGACCTCGAAACAGCAT \\
$2-5$ AS & CCAGGAAATTAGGAGACAGC & TGGCAGGGAGGAAGCAGGAG \\
MxA & GCATCCCACCCTCTATTACT & TGTCTTCAGTTCCTTTGTCC \\
TNF- $\alpha$ & ATGAGCACTGAAAGCATGATC & TCACAGGGCAATGATCCCAAAGTAGACCTGCCC \\
LT- $\beta$ & AAGCTGCCAGAGGAGGAGCC & TCCCGCTCGTCAGAAACGCC \\
Tax in HTLV-1-infected T cells & CCGGCGCTGCTCTCATCCCGGT & GGCCGAACATAGTCCCCCAGAG \\
Tax in JPX-9 cells & ATCGGCTCAGCTCTACAGTTCCT & ATTCGCTTGTAGGGAACATTGGT \\
$\beta$-actin & GTGGGGCGCCCCAGGCACCA & CTCCTTAATGTCACGCACGATTTC \\
GAPDH & GCCAAGGTCATCCATGACAACTTTGG & GCCTGCTTCACCACCTTCTTGATGTC \\
\end{tabular}

PBMC co-cultured with MMC-treated MT-2 cells expressed Tax mRNA (Fig. 3B). Furthermore, IRF-5 expression levels increased in these cells following induction of HTLV-1 gene. Noteworthy, IRF-5 expression was still detected in PBMC at 14 days after co-cultivation, which expressed Tax at a very low level (Fig. 3B). These results indicate that HTLV-1 infection induces the expression of IRF-5 in PBMC as well as T-cell line.

Direct effect of Tax on expression of IRF-5. Tax gene product is the primary viral transactivator protein that modulates the expression of both viral and cellular genes $(17,18)$. To test whether Tax directly induces IRF-5 expression, we used JPX-9, the Jurkat subline carrying Tax under the control of the metallothionein gene promoter (28). This cell line has been widely used to examine the effect of Tax on the expression of various cellular genes (28). The results are shown in Fig. 3C. Treatment of JPX-9 with $\mathrm{CdCl}_{2}$ rapidly induced Tax mRNA expression (panel 1). Similarly, Tax protein expression was induced within $24 \mathrm{~h}$ after addition of $\mathrm{CdCl}_{2}$ and reached a maximal level within $120 \mathrm{~h}$ (panel 7). Expression of the IRF-5 
A

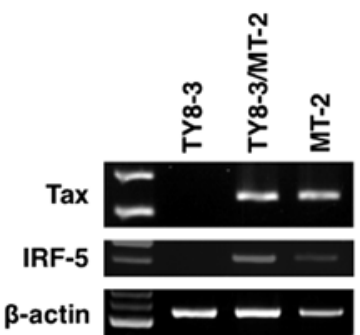

C

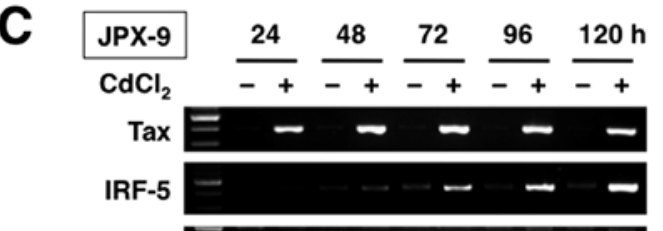

IRF-5 (V1N4) $=$

IRF-5 (V2) =

IRF-5 (V3)

$\beta$-actin $=-\infty \sim-\sigma-\sigma-\sigma-$

Tax

IRF-5

Actin
B
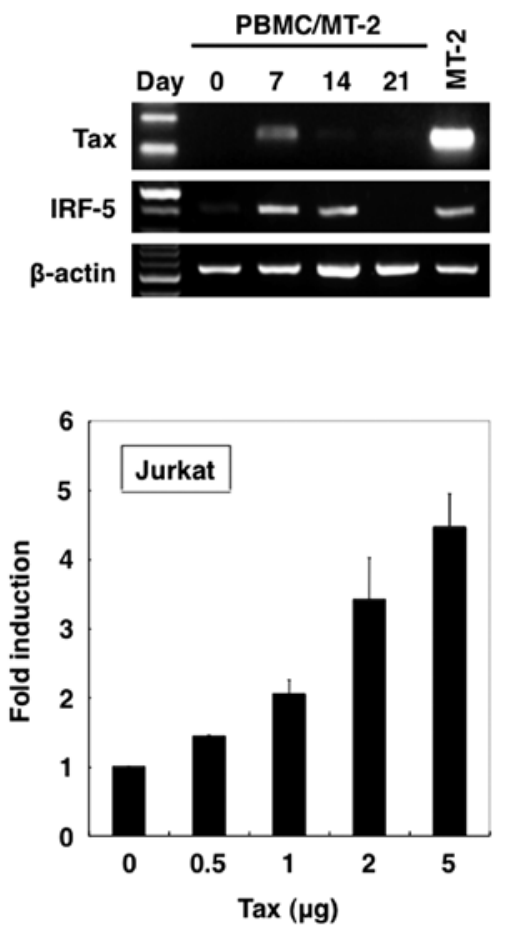

Figure 3. HTLV-1 infection and viral Tax expression induce IRF-5 expression. (A) RT-PCR analysis for mRNA expression of Tax and IRF-5 in TY8-3 and virally transformed TY8-3/MT-2 T cells. (B) Expression of Tax and IRF-5 in HTLV-1-infected PBMC. Normal PBMC were co-cultured with MMC-treated MT-2 cells. After co-cultivation, cells were harvested, and the expression of the indicated genes was analyzed by RT-PCR. (C) Induction of IRF-5 by Tax. JPX-9 cells were treated with $20 \mu \mathrm{M}$ of $\mathrm{CdCl}_{2}$ for 24-120 h. RT-PCR was carried out for the indicated genes (panels 1-6). Western blot analysis was also performed (panels 7-9). (D) Tax also regulates the P-V3 promoter. The P-V3 construct was co-transfected with increasing amounts of expression plasmid for Tax. In all transfection assays the levels of reporter firefly luciferase activity were normalized to a constant level of phRL-TK Renilla luciferase activity that served as an internal control. The results are presented as fold induction of luciferase activity of the P-V3 construct without Tax in Jurkat cells, which was considered 1. Data are mean \pm SD of three independent experiments.

gene was not detected at $24 \mathrm{~h}$ but became evident within $48 \mathrm{~h}$ after addition of $\mathrm{CdCl}_{2}$ (panel 2). A significant increase in the level of expression of IRF-5 protein (panel 8) was also noted; the protein level began to rise $96 \mathrm{~h}$ after the addition of $\mathrm{CdCl}_{2}$ with the maximal level observed at $120 \mathrm{~h}$.

We next examined IRF-5 isoform expression in $\mathrm{CdCl}_{2}$ treated and -untreated JPX-9 cells. As shown in Fig. $3 \mathrm{C}, \mathrm{CdCl}_{2}$ upregulated Ex1 V1-associated transcript levels (panel 3), whereas V2 transcript levels were not detected in treated or untreated samples (panel 4). In comparison, $\mathrm{CdCl}_{2}$ upregulated IRF-5 V3 transcript levels (panel 5).

We also examined the effects of Tax on IRF-5 isoform 3 promoter $(\mathrm{P}-\mathrm{V} 3)$. The activities of the reporter construct containing the entire $\mathrm{P}-\mathrm{V} 3$ region were analyzed in transient transfection assays in Jurkat cells (Fig. 3D). Co-transfection of the P-V3 promoter construct in Jurkat cells with Tax expression plasmid enhanced promoter activity in Tax dose-dependent manner. Considered collectively, the above results indicate that Tax can activate IRF-5 P-V3 promoter.

Type I IFN does not regulate IRF-5. Type I IFN is a wellknown transcriptional inducer of IRF-5 (10). However, its efficacy on IRF-5 induction in T cells is still unknown. Jurkat cells were exposed to IFN- $\alpha$ before RT-PCR. Unexpectedly, IFN- $\alpha$ failed to induce IRF-5 (Fig. 4A). However, treatment of these cells with IFN- $\alpha$ significantly increased the levels of other IFN-stimulated genes, $M x A$ and 2-5 AS.
IRF-5 targets TNF family cytokine genes. To assess the potential role of IRF-5 in initiating HTLV-1-infected cell-characteristic gene expression in T cells, Jurkat cells were transfected with control or IRF-5 expression plasmid. After $24 \mathrm{~h}$ of transfection, RT-PCR and immunoblotting were performed using specific primers, and anti-IRF-5 and anti-Myc antibodies to confirm transgene expression (Fig. 4B). In these experiments, changes in IRF-5-induced gene expression were analyzed by microarray analysis. The analysis showed significant enrichment of the up- and downregulated genes in IRF-5-transfected T cells. Table II shows genes that were upregulated by at least 5-fold in the presence of IRF-5. Previous studies reported that HTLV-1 infection induces T-cell activation and in vitro spontaneous lymphocyte proliferation, leading to the production of high levels of TNF- $\alpha$ in non-stimulated PBMC (43). Typical features of HTLV-1-infected T cells were recapitulated by IRF- 5 activity, such as upregulation of TNF- $\alpha$ and LT- $\beta$ (Table II and Fig. 4B).

Tax can also induce TNF- $\alpha$ expression. HTLV-1-infected T-cell lines constitutively secrete TNF- $\alpha$ (44). Furthermore, TNF- $\alpha$ expression is activated in the arthritic joints of Tax transgenic mice compared with the normal joints of non-transgenic mice (45). In the next set of experiments, we examined the effects of Tax on TNF- $\alpha$ expression in T cells. As expected, TNF- $\alpha$ was induced in Tax-expressing JPX-9 (Fig. 5A). Next, we used several T-cell lines to analyze the role of IRF-5 in the 


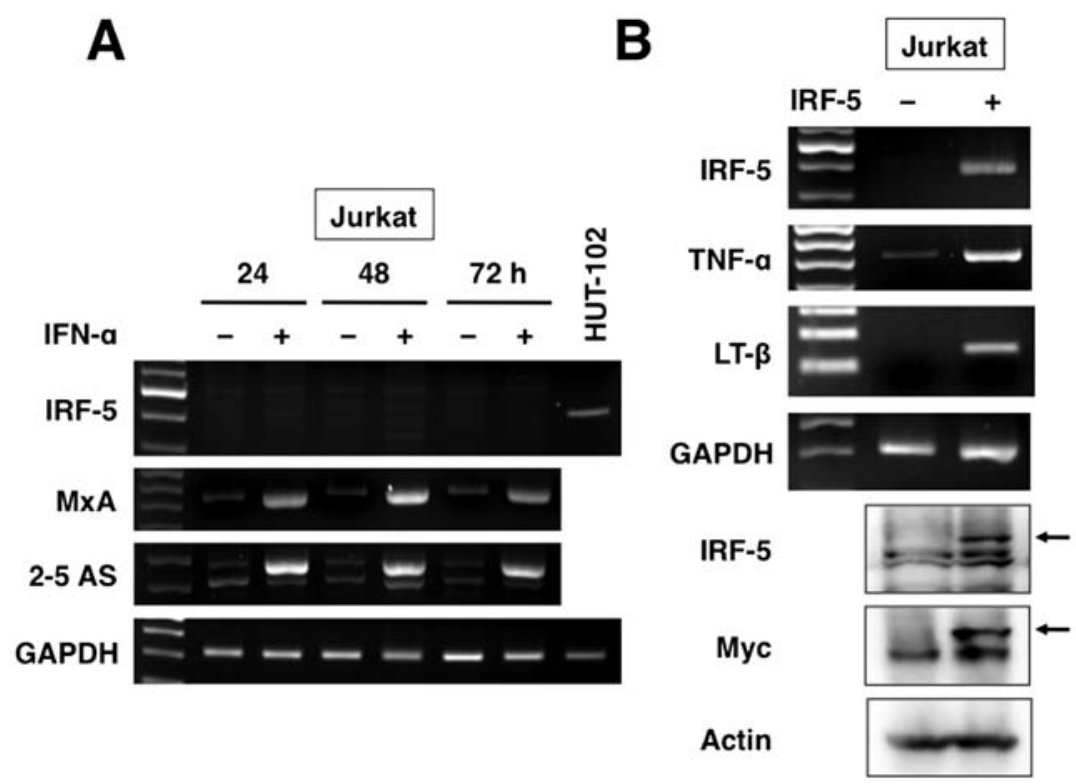

Figure 4. IFN- $\alpha$ does not induce IRF-5, and IRF-5 induces TNF family cytokines in T cells. (A) Jurkat cells were treated with IFN- $\alpha$ for the indicated time intervals, and subsequently analyzed by RT-PCR for the indicated IFN-regulatory genes. (B) IRF-5 induces the expression of TNF- $\alpha$ and LT- $\beta$ in T cells. Jurkat cells were transfected with control or Myc-tagged IRF-5 expression plasmid. After $24 \mathrm{~h}$, the expression levels of the indicated genes were determined by RT-PCR (panels 1-4). The level of Myc-tagged IRF-5 expression was also confirmed by immunoblotting with anti-IRF-5 (panel 5) and anti-Myc antibodies (panel 6). Left lane, empty vector control; right lane, Myc-tagged IRF-5 expressing Jurkat cells.

Table II. IRF-5-mediated genes with $\geq 5$ fold change.

\begin{tabular}{|c|c|c|c|}
\hline Symbol & Accession no. & Gene & Fold change \\
\hline IL17F & NM_052872 & Interleukin $17 \mathrm{~F}$ & 8.69 \\
\hline AZU1 & NM_001700 & Azurocidin 1 & 7.34 \\
\hline TNF & NM_000594 & Tumor necrosis factor & 7.24 \\
\hline PYY & NM_004160 & Peptide YY & 7.03 \\
\hline TEK & NM_000459 & TEK tyrosine kinase, endothelial & 6.82 \\
\hline LTB & NM_002341 & Lymphotoxin $\beta$ (TNF superfamily, member 3) & 5.84 \\
\hline SLC24A4 & NM_153646 & Solute carrier family 24 (sodium/potassium/calcium exchanger), member 4 & 5.76 \\
\hline ISPD & NM_001101426 & Isoprenoid synthase domain containing & 5.76 \\
\hline NRXN2 & NM_138732 & Neurexin 2 & 5.67 \\
\hline TMEM200A & NM_052913 & Transmembrane protein $200 \mathrm{~A}$ & 5.65 \\
\hline SFRP1 & NM_003012 & Secreted frizzled-related protein 1 & 5.47 \\
\hline KIR2DS4 & NM_012314 & Killer cell immunoglobulin-like receptor, two domains, short cytoplasmic tail, 4 & 5.42 \\
\hline SDCBP2 & NM_080489 & Syndecan binding protein (syntenin) 2 & 5.42 \\
\hline KLHDC7B & NM_138433 & Kelch domain containing 7B & 5.38 \\
\hline FAM176A & NM_032181 & Family with sequence similarity 176 , member A & 5.19 \\
\hline THADA & NM_001083953 & Thyroid adenoma associated & 5.18 \\
\hline GALNTL1 & NM_020692 & UDP-N-acetyl- $\alpha$-D-galactosamine (polypeptide $\mathrm{N}$-acetylgalactosaminyltransferase-like 1 ) & 5.10 \\
\hline CGA & NM_000735 & Glycoprotein hormones, $\alpha$ polypeptide & 5.02 \\
\hline OR10A7 & NM_001005280 & Olfactory receptor, family 10 , subfamily A, member 7 & 5.00 \\
\hline
\end{tabular}

expression of type I and II IFN, and TNF- $\alpha$. Compared to control T-cell lines, HTLV-1-infected T-cell lines expressed high levels of TNF- $\alpha$ (Fig. 5B). Similar to control T-cell lines, HTLV-1-infected T-cell lines also constitutively expressed IFN- $\alpha$ and $-\beta$. IFN- $\gamma$ expression was not associated with HTLV-1 infection. There was no correlation between IFN and Tax expression in HTLV-1-infected T-cell lines. The constitutive expression of TNF- $\alpha$ tended to be associated with IRF-5 or Tax expression.

\section{Discussion}

IRF-5 mRNA expression has been detected in B cells, dendritic cells, monocytes and natural killer cells but not in T cells (30). 


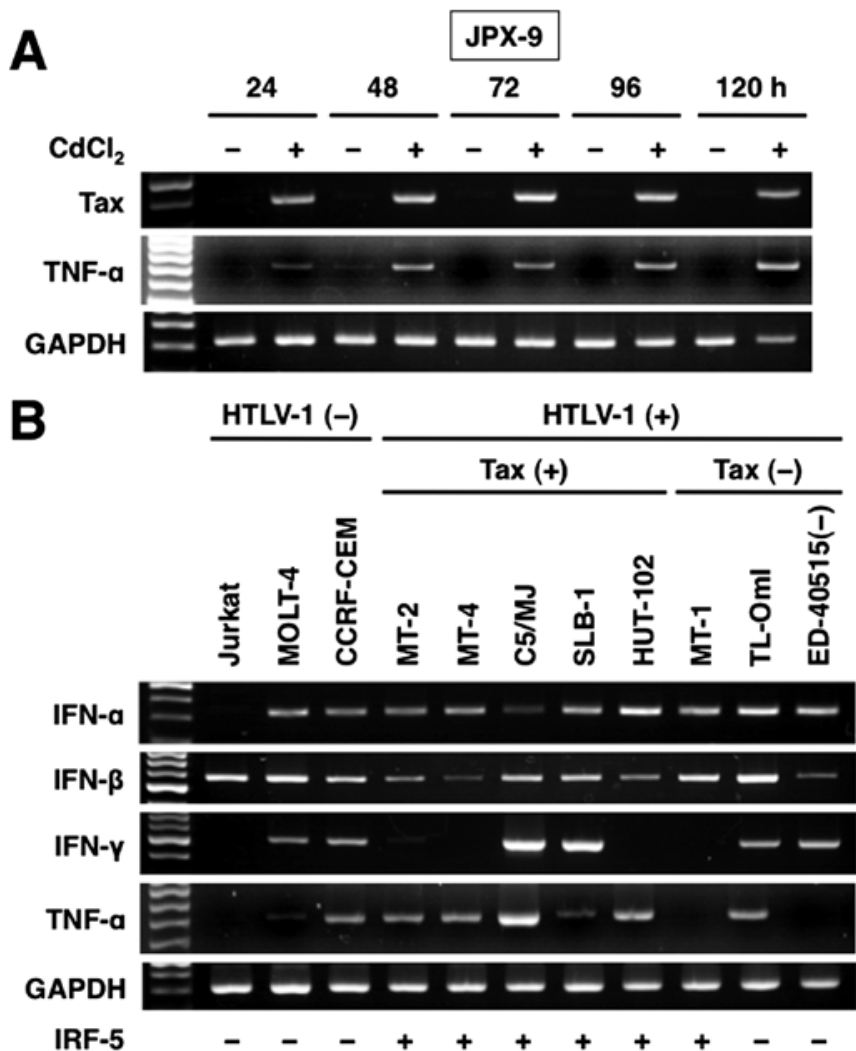

Figure 5. Induction of TNF- $\alpha$ by Tax, and expression of IFN and TNF- $\alpha$ in several T-cell lines. (A) Tax induces TNF- $\alpha$ expression. RT-PCR was carried out for TNF- $\alpha$ in JPX-9 cells treated with $\mathrm{CdCl}_{2}$. (B) RT-PCR analysis for expression of IFN and TNF- $\alpha$ in the T-cell lines.

In the present study, we demonstrated that $\mathrm{T}$ cells acquire high levels of IRF-5 expression during HTLV-1 infection. Our results are consistent with those of oligonucleotide microarray reported by Baba et al (46), who identified IRF-5 to be one of the genes that were upregulated more than 40 -fold in three HTLV-1-infected T-cell lines, including MT-2, compared with uninfected T-cell line MOLT-4. Our results also showed that the oncoprotein Tax activated the IRF-5 V3 promoter, and that IRF-5 was expressed in ATL cells infiltrating the lymph nodes and skin. Whether the expression of IRF-5 is present in PBMC samples from patients with ATL containing leukemic cells (which do not express Tax protein) was not examined and thus remains a very interesting question. Importantly, ATL-derived MT-1 cells, which also do not express Tax protein, expressed high levels of IRF-5 protein. These results suggest that Tax-independent IRF-5 expression mechanisms may also exist in ATL cells.

To establish the effects of endogenous IRF-5 in HTLV-1infected $\mathrm{T}$ cells, we silenced its expression using the siRNA in HUT-102 cells (data not shown). Although IRF-5 silencing was confirmed by RT-PCR and western blot analysis, reduced IRF-5 expression did not affect cell growth (data not shown). In this study, the role of IRF-5 in T cells was investigated using IRF-5-expressing Jurkat cells and cDNA array technology. The results showed that IRF-5 expression does not affect the expression of cell growth- and apoptosis-related genes (data not shown). Furthermore, IRF-5 overexpression did not increase the proliferation of Jurkat cells (data not shown). These studies confirmed that IRF-5 does not directly modulate cell growth However, the exact IRF-5 function in cell proliferation needs to be further investigated using IRF-5 stable transfectants.

IRF-5 exists in multiple alternatively spliced isoforms that are expressed in a cell type-specific manner (30). The present results demonstrated that the induction of IRF-5 expression by Tax is isoform-specific. Tax-positive HTLV-1-infected T-cell lines specifically upregulated Ex1V3 transcripts, and the Ex1V3-specific transcripts were upregulated by Tax expression. Indeed, promoter reporter assays demonstrated that Tax enhanced P-V3 promoter activity.

IRF-5 is a central mediator that controls the expression of type I IFN (2). However, there was no link between IRF-5 and type I IFN expression in HTLV-1-infected T cell lines. In addition, IFN- $\alpha$ did not upregulate IRF-5 in Jurkat T cells. Although this finding does not completely exclude IFN- $\alpha$ induced IRF-5 expression in primary peripheral $\mathrm{T}$ cells, the expression of type I IFN seems to be independent of IRF-5 in HTLV-1-infected T cells. On the contrary, IRF-5 expression induced other TNF family genes. TNF- $\alpha$ is a major cytokine involved in the promotion of inflammatory responses, and it plays a crucial role in the pathogenesis of various inflammatory, autoimmune and malignant diseases (47). Genetic polymorphisms leading to increased TNF- $\alpha$ production enhance susceptibility to not only ATL (48), but also to uveitis, another HTLV-1-related disease (49). TNF- $\alpha$ is suggested to contribute to the high levels of organ infiltration by leukemic cells in ATL (50). Tax can activate mouse TNF- $\alpha$ promoter through nuclear factor- $\mathrm{\kappa B}(\mathrm{NF}-\mathrm{\kappa B})$ activation (51). Furthermore, IRF-5 can specifically interact with NF- $\kappa B$ RelA, and sustain TNF- $\alpha$ secretion in human dendritic cells (52). These findings suggest that Tax/NF- $\mathrm{KB} / \mathrm{IRF}-5$ may cooperate in HTLV-1-induced TNF- $\alpha$ promoter activation.

In summary, the present study indicates that high-level IRF-5 expression is specific to HTLV-1-infected T cells. The main function of IRF-5 in ATL and other HTLV-1-related disease should be investigated.

\section{Acknowledgements}

The authors thank Keisuke Kidoguchi and Takano Ohta for their excellent assistance. We also thank Drs Martin Schmidt, Kayoko Matsumoto and Yuetsu Tanaka for providing expression vectors for IRF-5 and Tax, and Tax antibody, as well as Dr Masataka Nakamura for providing JPX-9, Dr Michiyuki Maeda for providing ED-40515(-), and Fujisaki Cell Center, Hayashibara Biochemical Laboratories, Inc. (Okayama, Japan) for providing C5/MJ, HUT-102 and MT-1. Recombinant human IL-2 was kindly provided by Takeda Pharmaceutical Company Ltd. (Osaka, Japan). This work was supported in part by JSPS KAKENHI grant numbers 90542358 and 25461428 .

\section{References}

1. Battistini A: Interferon regulatory factors in hematopoietic cell differentiation and immune regulation. J Interferon Cytokine Res 29: 765-780, 2009.

2. Barnes BJ, Moore PA and Pitha PM: Virus-specific activation of a novel interferon regulatory factor, IRF-5, results in the induction of distinct interferon $\alpha$ genes. J Biol Chem 276: 23382-23390, 2001. 
3. Takaoka A, Tamura T and Taniguchi T: Interferon regulatory factor family of transcription factors and regulation of oncogenesis. Cancer Sci 99: 467-478, 2008.

4. Yanai H, Chen H-M, Inuzuka T, Kondo S, Mak TW, Takaoka A, Honda $\mathrm{K}$ and Taniguchi T: Role of IFN regulatory factor 5 transcription factor in antiviral immunity and tumor suppression. Proc Natl Acad Sci USA 104: 3402-3407, 2007.

5. Tamura T, Yanai H, Savitsky D and Taniguchi T: The IRF family transcription factors in immunity and oncogenesis. Annu Rev Immunol 26: 535-584, 2008.

6. Hu G and Barnes BJ: IRF-5 is a mediator of the death receptorinduced apoptotic signaling pathway. J Biol Chem 284: 2767-2777, 2009.

7. Graham RR,Kozyrev SV, BaechlerEC, Reddy MVPL,Plenge RM, Bauer JW, Ortmann WA, Koeuth T, González Escribano MF; Argentine and Spanish Collaborative Groups, et al: A common haplotype of interferon regulatory factor 5 (IRF5) regulates splicing and expression and is associated with increased risk of systemic lupus erythematosus. Nat Genet 38: 550-555, 2006.

8. Dawidowicz K, Allanore Y, Guedj M, Pierlot C, Bombardieri S, Balsa A, Westhovens R, Barrera P, Alves $\mathrm{H}$, Teixeira $\mathrm{VH}$, et al; ECRAF: The interferon regulatory factor 5 gene confers susceptibility to rheumatoid arthritis and influences its erosive phenotype. Ann Rheum Dis 70: 117-121, 2011.

9. Mori T, Anazawa Y, Iiizumi M, Fukuda S, Nakamura Y and Arakawa H: Identification of the interferon regulatory factor 5 gene $(I R F-5)$ as a direct target for p53. Oncogene 21: 2914-2918, 2002.

10. Barnes BJ, Kellum MJ, Pinder KE, Frisancho JA and Pitha PM: Interferon regulatory factor 5, a novel mediator of cell cycle arrest and cell death. Cancer Res 63: 6424-6431, 2003.

11. Shin SH, Kim B-H, Jang J-J, Suh KS and Kang GH: Identification of novel methylation markers in hepatocellular carcinoma using a methylation array. J Korean Med Sci 25: 1152-1159, 2010.

12. Yamashita M, Toyota M, Suzuki H, Nojima M, Yamamoto E, Kamimae S, Watanabe Y, Kai M, Akashi H, Maruyama R, et al: DNA methylation of interferon regulatory factors in gastric cancer and noncancerous gastric mucosae. Cancer Sci 101: 1708-1716, 2010.

13. Massimino M, Vigneri P, Fallica M, Fidilio A, Aloisi A, Frasca F and Manzella L: IRF5 promotes the proliferation of human thyroid cancer cells. Mol Cancer 11: 21, 2012.

14. Kreher S, Bouhlel MA, Cauchy P, Lamprecht B, Li S, Grau M, Hummel F, Köchert K, Anagnostopoulos I, Jöhrens K, et al: Mapping of transcription factor motifs in active chromatin identifies IRF5 as key regulator in classical Hodgkin lymphoma. Proc Natl Acad Sci USA 111: E4513-E4522, 2014.

15. Kannian P and Green PL: Human T lymphotropic virus type 1 (HTLV-1): Molecular biology and oncogenesis. Viruses 2: 2037-2077, 2010.

16. Hasunuma T, Sumida T and Nishioka K: Human T cell leukemia virus type-I and rheumatoid arthritis. Int Rev Immunol 17: 291-307, 1998

17. Grassmann R, Aboud M and Jeang K-T: Molecular mechanisms of cellular transformation by HTLV-1 Tax. Oncogene 24: 5976-5985, 2005.

18. Currer R, Van Duyne R, Jaworski E, Guendel I, Sampey G, Das R, Narayanan A and Kashanchi F: HTLV tax: A fascinating multifunctional co-regulator of viral and cellular pathways. Front Microbiol 3: 406, 2012.

19. Ohsugi T: A transgenic mouse model of human T cell leukemia virus type 1-associated diseases. Front Microbiol 4: 49, 2013.

20. Suzuki S, Zhou Y, Refaat A, Takasaki I, Koizumi K, Yamaoka S, Tabuchi Y, Saiki I and Sakurai H: Human T cell lymphotropic virus 1 manipulates interferon regulatory signals by controlling the TAK1-IRF3 and IRF4 pathways. J Biol Chem 285: 4441-4446, 2010.

21. Sharma S, Mamane Y, Grandvaux N, Bartlett J, Petropoulos L, Lin $\mathrm{R}$ and Hiscott $\mathrm{J}$ : Activation and regulation of interferon regulatory factor 4 in HTLV type 1-infected T lymphocytes. AIDS Res Hum Retroviruses 16: 1613-1622, 2000.

22. Tsuboi K, Iida S, Inagaki $\mathrm{H}$, Kato M, Hayami Y, Hanamura I, Miura K, Harada S, Kikuchi M, Komatsu H, et al: MUM1/IRF4 expression as a frequent event in mature lymphoid malignancies. Leukemia 14: 449-456, 2000

23. Imaizumi Y,Kohno T, Yamada Y,Ikeda S, Tanaka Y, Tomonaga M and Matsuyama T: Possible involvement of interferon regulatory factor 4 (IRF4) in a clinical subtype of adult T-cell leukemia. Jpn J Cancer Res 92: 1284-1292, 2001.
24. Ramos JC, Ruiz P Jr, Ratner L, Reis IM, Brites C, Pedroso C, Byrne GE Jr, Toomey NL, Andela V, Harhaj EW, et al: IRF-4 and c-Rel expression in antiviral-resistant adult T-cell leukemia/ lymphoma. Blood 109: 3060-3068, 2007.

25. Yamagata T, Nishida J, Tanaka S, Sakai R, Mitani K, Yoshida M, Taniguchi T, Yazaki Y and Hirai H: A novel interferon regulatory factor family transcription factor, ICSAT/Pip/LSIRF, that negatively regulates the activity of interferon-regulated genes. Mol Cell Biol 16: 1283-1294, 1996.

26. Sharma S, Grandvaux N, Mamane Y, Genin P, Azimi N, Waldmann T and Hiscott J: Regulation of IFN regulatory factor 4 expression in human $\mathrm{T}$ cell leukemia virus-I-transformed T cells. J Immunol 169: 3120-3130, 2002

27. Grumont RJ and Gerondakis S: Rel induces interferon regulatory factor $4(I R F-4)$ expression in lymphocytes: Modulation of interferon-regulated gene expression by rel/nuclear factor $\mathrm{\kappa B}$. J Exp Med 191: 1281-1292, 2000.

28. Ohtani K, Nakamura M, Saito S, Nagata K, Sugamura K and Hinuma Y: Electroporation: Application to human lymphoid cell lines for stable introduction of a transactivator gene of human T-cell leukemia virus type I. Nucleic Acids Res 17: 1589-1604, 1989.

29. Yoshida T, Miyagawa E, Yamaguchi K, Kobayashi S, Takahashi Y, Yamashita A, Miura H, Itoyama Y and Yamamoto N: IL-2 independent transformation of a unique human T cell line, TY8-3, and its subclones by HTLV-I and -II. Int J Cancer 91: 99-108, 2001.

30. Mancl ME, Hu G, Sangster-Guity N, Olshalsky SL, Hoops K, Fitzgerald-Bocarsly P, Pitha PM, Pinder K and Barnes BJ: Two discrete promoters regulate the alternatively spliced human interferon regulatory factor-5 isoforms. Multiple isoforms with distinct cell type-specific expression, localization, regulation, and function. J Biol Chem 280: 21078-21090, 2005.

31. Saikh KU, Lee JS, Kissner TL, Dyas B and Ulrich RG: Toll-like receptor and cytokine expression patterns of $\mathrm{CD}^{+} 6^{+} \mathrm{T}$ cells are similar to natural killer cells in response to infection with Venezuelan equine encephalitis virus replicons. J Infect Dis 188: $1562-1570,2003$

32. Iki S, Yokota S, Okabayashi T, Yokosawa N, Nagata K and Fujii N: Serum-dependent expression of promyelocytic leukemia protein suppresses propagation of influenza virus. Virology 343: $106-115,2005$

33. Yokosawa N, Kubota T and Fujii N: Poor induction of interferoninduced 2',5'-oligoadenylate synthetase (2-5 AS) in cells persistently infected with mumps virus is caused by decrease of STAT-1 $\alpha$. Arch Virol 143: 1985-1992, 1998.

34. Brenner CA, Tam AW, Nelson PA, Engleman EG, Suzuki N, Fry KE and Larrick JW: Message amplification phenotyping (MAPPing): A technique to simultaneously measure multiple mRNAs from small numbers of cells. Biotechniques 7: 1096-1103, 1989.

35. Subrata LS, Lowes KN, Olynyk JK, Yeoh GCT, Quail EA and Abraham LJ: Hepatic expression of the tumor necrosis factor family member lymphotoxin- $\beta$ is regulated by interleukin (IL)- 6 and IL-1 $\beta$ : Transcriptional control mechanisms in oval cells and hepatoma cell lines. Liver Int 25: 633-646, 2005.

36. Hieshima K, Nagakubo D, Nakayama T, Shirakawa AK, Jin Z and Yoshie O: Tax-inducible production of $\mathrm{CC}$ chemokine ligand 22 by human $\mathrm{T}$ cell leukemia virus type 1 (HTLV-1)-infected T cells promotes preferential transmission of HTLV-1 to CCR4expressing CD4 ${ }^{+}$T cells. J Immunol 180: 931-939, 2008.

37. Mori N and Prager D: Transactivation of the interleukin- $1 \alpha$ promoter by human T-cell leukemia virus type I and type II Tax proteins. Blood 87: 3410-3417, 1996.

38. Ishikawa C, Kawakami H, Uchihara J-N, Senba M and Mori N: CD69 overexpression by human T-cell leukemia virus type 1 Tax transactivation. Biochim Biophys Acta 1833: 1542-1552, 2013.

39. Suzuki K, Bose P, Leong-Quong RY, Fujita DJ and Riabowol K: REAP: A two minute cell fractionation method. BMC Res Notes 3: $294,2010$.

40. Tanaka Y, Yoshida A, Takayama Y, Tsujimoto H, Tsujimoto A, Hayami M and Tozawa H: Heterogeneity of antigen molecules recognized by anti-tax 1 monoclonal antibody Lt- 4 in cell lines bearing human $\mathrm{T}$ cell leukemia virus type I and related retroviruses. Jpn J Cancer Res 81: 225-231, 1990.

41. Matsumoto K, Shibata H, Fujisawa J-I, Inoue H, Hakura A, Tsukahara T and Fujii M: Human T-cell leukemia virus type 1 Tax protein transforms rat fibroblasts via two distinct pathways. J Virol 71: 4445-4451, 1997. 
42. Kimura R, Ishikawa C, Rokkaku T, Janknecht R and Mori N Phosphorylated c-Jun and Fra-1 induce matrix metalloproteinase-1 and thereby regulate invasion activity of 143B osteosarcoma cells. Biochim Biophys Acta 1813: 1543-1553, 2011.

43. Santos SB, Porto AF, Muniz AL, de Jesus AR, Magalhães E, Melo A, Dutra WO, Gollob KJ and Carvalho EM: Exacerbated inflammatory cellular immune response characteristics of HAM/TSP is observed in a large proportion of HTLV-I asymptomatic carriers. BMC Infect Dis 4: 7,2004.

44. Tschachler E, Robert-Guroff M, Gallo RC and Reitz MS Jr: Human T-lymphotropic virus I-infected T cells constitutively express lymphotoxin in vitro. Blood 73: 194-201, 1989.

45. Iwakura Y, Saijo S, Kioka Y, Nakayama-Yamada J, Itagaki K, Tosu M, Asano M, Kanai Y and Kakimoto K: Autoimmunity induction by human $\mathrm{T}$ cell leukemia virus type 1 in transgenic mice that develop chronic inflammatory arthropathy resembling rheumatoid arthritis in humans. J Immunol 155: 1588-1598, 1995.

46. Baba M, Okamoto M, Hamasaki T, Horai S, Wang X, Ito $Y$, Suda Y and Arima N: Highly enhanced expression of CD70 on human T-lymphotropic virus type 1-carrying T-cell lines and adult T-cell leukemia cells. J Virol 82: 3843-3852, 2008.

47. Bazzoni F and Beutler B: The tumor necrosis factor ligand and receptor families. N Engl J Med 334: 1717-1725, 1996.
48. Tsukasaki K, Miller CW, Kubota T, Takeuchi S, Fujimoto T, Ikeda S, Tomonaga M and Koeffler HP: Tumor necrosis factor $\alpha$ polymorphism associated with increased susceptibility to development of adult T-cell leukemia/lymphoma in human T-lymphotropic virus type 1 carriers. Cancer Res 61: 3770-3774, 2001.

49. Seki N, Yamaguchi K, Yamada A, Kamizono S, Sugita S, Taguchi C, Matsuoka M, Matsumoto H, Nishizaka S, Itoh K, et al: Polymorphism of the 5'-flanking region of the tumor necrosis factor (TNF)- $\alpha$ gene and susceptibility to human T-cell lymphotropic virus type I (HTLV-I) uveitis. J Infect Dis 180: 880-883, 1999.

50. Watters KM, Dean J, Hasegawa H, Sawa H, Hall W and Sheehy N: Cytokine and growth factor expression by HTLV-1 Lck-tax transgenic cells in SCID mice. AIDS Res Hum Retroviruses 26: 593-603, 2010.

51. Albrecht $\mathrm{H}$, Shakhov AN and Jongeneel CV: trans activation of the tumor necrosis factor alpha promoter by the human T-cell leukemia virus type I Tax 1 protein. J Virol 66: 6191-6193, 1992.

52. Krausgruber T, Saliba D, Ryzhakov G, Lanfrancotti A, Blazek K and Udalova IA: IRF5 is required for late-phase TNF secretion by human dendritic cells. Blood 115: 4421-4430, 2010. 